\title{
Prevention of postoperative wound infections - what would be worthwhile, and what might be done?
}

\author{
Geeta Mehta ${ }^{1}$, Ulrika Ransjö ${ }^{2}$ \\ ${ }^{1}$ Delhi, India; ${ }^{2}$ Uppsala, Sweden \\ doi:10.3396/ijic.V4i1.007.08
}

Postoperative wound infections or surgical site infections (SSI) have caused serious problems ever since surgery began. Without preventive efforts, at least 3\% of patients undergoing clean surgery and up to $30 \%$ of those undergoing contaminated or dirty surgery develop SSI. ${ }^{1}$ The economic impact of SSI is great from several aspects. The patient pays, through loss of income and through insurance payments if applicable. The hospital pays for salaries to nursing staff, doctors and ancillary staff; for disposable and reusable equipment; for drugs; for disinfection and sterilization; etc. The community pays, or cannot afford to pay, for loss of productivity and for the running of hospitals. The actual sizes of these costs are difficult to estimate, even though the cost-benefit ratios can be modelled. ${ }^{2}$

Deep SSI originates in the operating theatre, but the sources of infection can be the patient, the theatre environment or the operating room staff. Superficial wound infection may also originate from the ward environment, pre- or postoperatively. The routes of transmission can be several: the patient's own colonised or infected skin and internal organs, contaminated instruments, the hands of surgical and anaesthesiology staff, and airborne from the skin of staff to mention a few. Prevention of SSI needs to address all these sources of infection.

Prevention of surgical site infection (SSI) is an important issue in both high and low income countries. Printed material and references for infection prevention are available to affluent health care facilities, but considerably less exists for settings with limited resources. It is difficult for practitioners in such settings to prioritize choices of activities and materials.

\section{How to evaluate routines for prevention} of postoperative wound infections

Methods of prevention can be divided into four categories:

- Established methods for which there is adequate bacteriological and/or clinical evidence

- Provisionally established methods that need further evaluation but can be provisionally recommended

- Rational methods that seem desirable but cannot be evaluated

- Rituals that are traditionally observed but probably unnecessary and sometimes potentially harmful 


\section{Recommendations for the prevention of SSI should be evidence based}

Evidence for lowering of infection rates in clean surgery requires very large studies, which are costly and difficult to conduct. Scientific studies, or what we nowadays like to call evidence-based, sometimes, but by no means always, substantiate the recommendations in national guidelines of many countries.

Large patient groups would be needed to prove that a reduction in SSI rates in clean surgery can be achieved with a given method. Such studies would have to be protracted for many years if performed in one centre, and costly, as well as hard to organize, as multi-centre studies. The studies can be randomized, and sometimes evaluator blinded, but it is only in studies of medication and such that they can be double- blinded: usually it is very obvious which skin disinfectant, clothing, surgical technique, or type of wound dressing is being used. Surrogate markers such as wound cultures or length of stay are often used.

The operating theatres are now a market for medical products, sometimes at great cost. The products have to comply with certain standards if they are to be sold in the US or Europe. However, manufacturers sometimes compete by setting the performance levels of their products way above the standards. The International Federation of Infection Control (IFIC) attempts to provide guidelines that are appropriate and, as much as possible, cost effective.

\section{Recommendations}

Based on available evidence, extensive recommendations have been made. ${ }^{2}$ These include the following, as quoted from the IFIC Basic Concepts in Infection Control (minimal requirements in bold script):

\section{The patient}

1. Identify and treat all infections before elective operations.

2. Keep preoperative hospital stay to a minimum.

3. Do not remove hair preoperatively unless the hair at or around the incision site will interfere with the operation. If considered essential, remove hair immediately before the operation with a non invasive procedure, e.g., clipper.
4. Good control of diabetes is essential in the perioperative period.

5. Administer prophylactic antibiotics when indicated, according to established criteria and local policy.

6. Use an antiseptic agent for skin preparation immediately prior to the operation.

\section{The surgical team}

1. Perform a preoperative surgical scrub for at least 2-4 minutes using an appropriate antiseptic. Do not use a brush. Remove debris underneath the fingernails using a nail cleaner before the first procedure in the morning.

2. Personnel with draining skin lesions must be excluded until the lesions are fully resolved.

3. Limit the duration of the procedure as much as possible.

4. Wear sterile gloves. Put gloves on after donning a sterile gown. Use sterile water repellent surgical gowns and drapes. Wear a surgical mask and a cap or hood to fully cover hair.

5. Adhere to principles of asepsis when performing interventions and invasive procedures in the operating room, e.g., placing central venous, spinal or epidural anaesthesia catheters or when dispensing and administering intravenous drugs.

6. Handle tissue gently, maintain effective homeostasis, minimize devitalized tissue and foreign bodies (e.g., sutures, charred tissues, necrotic debris), and eradicate dead space at the surgical site.

7. Use closed suction drains. Place a drain through a separate incision distant from the operative incision. Remove it as soon as possible.

\section{The operating room environment}

1. Maintain positive pressure ventilation in the operating room with respect to the corridors and adjacent areas. Twenty air changes per hour are recommended. Filter all air, recirculated and fresh.

2. Keep operating room doors closed except as needed for passage of equipment, personnel, and the patient.

3. Restrict the number of personnel entering the operating room to necessary personnel only, and restrict their movement.

4. Sterilize all surgical instruments with validated methods. Do not use flash sterilization routinely. 
5. Do not perform special cleaning or closing of operating rooms after contaminated or dirty operations.

6. Do not use over-shoes and tacky mats at the entrance to the operating room suite.

\section{Postoperatively}

1. Do not touch the wound unless it is necessary.

2. Have an on-going surveillance system for SSI using standard definitions and risk classifications.

3. Perform post-discharge surveillance for patients with ambulatory surgery or a short hospital stay.

\section{Compliance to available guidelines}

Even if guidelines are to some extent evidence based, we have very little data on compliance: what resources are available in terms of buildings, equipment or personnel and are the resources properly used? The outcome might be measured in terms of outbreaks or incidence of postoperative wound infections, but resources for surveillance are rarely available. One of the authors (GM) therefore performed a survey of infection control (IC) practices concerning the prevention of SSI among infection control professionals visiting IC conferences. The data will be published in full elsewhere, but we thought it important to share some of them with the IFIC audience.

Over 50 IC professionals were interviewed; 31 came from low income countries (LIC) and 23 from middle income countries (MIC). The questionnaire was based on the Healthcare Infection Control Practices Advisory Committee's recommendations $^{2}$ and contained 54 questions. The questionnaires were administered in group settings, at times through an individual interview or over the telephone.

The respondent was required to score each recommendation: to what extent was compliance to the recommendation "possible" and given it was possible then how "likely" was it that the recommendation would be complied with. "Possible" was defined as having the basic supplies, equipment, and facilities necessary for the task. "Likely" was defined as having the basic supplies and necessary systems and also having the human resources, education, knowledge and attitudes in place for accomplishing the task. Some areas are highlighted below.
- Preoperative hair removal was possible and likely to be performed according to guidelines in $90 \%$ of both LIC and MIC.

- Antimicrobial prophylaxis administered according to current guidelines was fully possible in both groups, but only 16 of $31 \mathrm{LIC}$ responders thought it likely that this would actually materialize $(\mathrm{p}=0.01)$.

- Antiseptic cleaning of the operating site was fully possible and likely in both income classes.

- To use surgical gowns and drapes that are barriers to liquid penetration was possible in 19 of 23 MIC but only in 17 of 31 LIC (p 0.034 in MantelHaenzel test).

- Closed suction drains were used in practically all hospitals.

- To keep the doors of the operating theatre closed during surgery is low cost, and yet 8 of 31 low income IC professionals thought it unlikely that this would be adhered to.

- Sterilization of instruments was performed properly in all MIC and in 26 of $31 \mathrm{LIC}(\mathrm{p}=0.045)$.

- Special cleaning after dirty operation was likely to be performed in 4 of $23 \mathrm{MIC}$ but in 20 of 31 LIC $(p=0.001)$ despite the recommendations

- Shoe covers and tacky mats were rarely used

\section{Comments and discussion}

Antimicrobial prophylaxis is probably the single most cost-effective measure against SSI. Despite this, and even though it was fully possible to administer the prophylaxis correctly, the IC professionals interviewed from low income countries thought it less than 50\% likely that this would happen in their hospitals. This and other simple measures were significantly less likely to be undertaken in low income countries, despite the fact that they were fully possible. Rituals like special cleaning after dirty operations and the use of tacky mats, etc, were more common in low income countries.

Overall, several significant differences in the responses were observed between the MIC and LIC participants. For most recommendations, respondents from MIC showed a higher compliance level indicating that they had the required infrastructure (assessed by the "possible" responses) and the capacity (assessed by the 'likely' responses) as compared to the LIC. 
Moreover, the difference in compliance level was not entirely dependent on the lack of infrastructure, the LIC were less likely to comply with a recommendation even if they had the requisite infrastructure. This can be attributed to a lack of capacity to utilize the infrastructure appropriately. The barriers highlighted by the respondents were:

a) lack of training and education

b) existing outdated policies and practices such as those for pre-operative removal of hair, policies for special cleaning and fumigation of the operating room and antibiotic prophylaxis policy

c) lack of administrative will

d) misconceptions such as considering antibiotics and antiseptics as a panacea for infections and

e) careless attitude.
These are harsh words, but also provide a basis for revision of guidelines and policies, which would then be used for education and audit. The IFIC Basic Concepts in Infection Control could form a basis both for revision and for training, which is just what they were developed for.

\section{References}

1. Graves N, Halton K, Lairson D. Economics and Preventing Hospital-Acquired Infection: Broadening the Perspective. Infect Control Hosp Epidemiol 2007; 28: 178-184.

2. Mangram, AJ, Horan TC, Pearson ML, Silver LC, Jarvis WR . Guideline for Prevention of Surgical Site Infection, 1999. The Hospital Infection Control Practices Advisory Committee. Infect Control Hosp Epidemiol 1999; 20: 247. 